\title{
Sleep Overlap Syndrome and Association with Other Medical Disorders
}

\author{
Varsha Taskar ${ }^{1}$, Tenzing Phanthok ${ }^{2}$, Crystal Duran $^{3}$ \\ International Journal of Head and Neck Surgery (2019): 10.5005/jp-journals-10001-1372
}

\section{Case Description}

A 55-year-old male with end-stage renal disease on hemodialysis presents with excessive daytime sleepiness and fatigue. Patient reports compliance with his dialysis sessions. He typically goes to bed at $11 \mathrm{pm}$, takes up to an hour to fall asleep, and wakes up at $6 \mathrm{am}$. Sometimes he wakes up during the night with leg cramps and may have to get up and walk around his room to alleviate the cramping. Wife reports loud snoring and intermittent periods during which the patient stops breathing. He is capable of self care, but has reported lack of interest and energy and often prefers to stay at home. He is noted to fall asleep while watching the news. He does not drink alcohol.

\section{Diagnostic Considerations for Sleep Disruption}

- Sleep onset insomnia - daytime sleeping or while on dialysis may disrupt their sleep/wake cycle

- Restless leg syndrome - circadian preponderance and relief with movement

- Complex sleep apnea - obstructive sleep apnea related to body habitus and central component related to fluid overload, congestive heart failure

- Excessive daytime sleepiness versus metabolic causes of fatigue. Sleep and medical disorders have a complex interdependency. While sleep is ultimately affected in most medical disorders, treatment of the sleep or medical disorder can also positively impact the course of the illness. Quality of life indicators can be used to assess the impact of the disorder on sleep since that frequently unmasks the severity and stability of the chronic illness and ultimately the individual's capacity to cope. For example, asthma may be controlled in the daytime but may still have nocturnal symptoms suggesting room for optimization. Both excessive daytime sleepiness and insomnia increase with age and may destabilize a stable chronic illness or complicate recovery from an acute one.

\section{Mechanism of sleep disorders in medical illness}

- Effect of metabolic disturbances on the respiratory neurons and respiratory drive causing sleep-related breathing disorders

- Adverse effect of medications and metabolites causing difficulty falling and staying asleep

- Effect on the sleep/wake cycle

- Effect on neuromuscular system causing spasms, pain, cramps, and leg movement activity.

The impact of sleep disordered breathing such as in obstructive sleep apnea (OSA) is defined by recurrent upper airway obstruction resulting in episodic hypopnea or apnea and interruption of normal sleep resulting in a nonrestorative sleep. Sleep-related
${ }^{1-3}$ Division of Pulmonology, Department of Internal Medicine, Augusta University Medical Center, Augusta, Georgia, USA

Corresponding Author: Varsha Taskar, Division of Pulmonology, Department of Internal Medicine, Augusta University Medical Center, Augusta, Georgia, USA, e-mail:VTASKAR@augusta.edu

How to cite this article: Taskar V, Phanthok T, Duran C. Sleep Overlap Syndrome and Association with Other Medical Disorders. Int J Head Neck Surg 2019;10(3):55-56.

Source of support: Nil

Conflict of interest: None

breathing disorder thus results in sleep fragmentation causing major downstream multisystem consequences.

- Chronic obstructive pulmonary disease (COPD): COPD and OSA when occurring simultaneously is referred to as 'overlap syndrome,' as first described by Flenley in $1985 .{ }^{1}$ OSA can result in significant hypoxia during sleep that can result in pulmonary and systemic hypertension over time. The reduced oxygen saturation in COPD is worsened in the setting of OSA. Patients with overlap syndrome have a higher risk of morbidity and mortality compared to individuals with just COPD or OSA. In COPD patients with untreated OSA, there is an increased risk of mortality and hospitalization for acute exacerbations. Treatment includes recognizing overlap syndrome and obtaining polysomnograms on these individuals. A common approach includes the use of continuous airway positive pressure (CPAP) often in combination with supplemental oxygen.

- Asthma: It is a chronic inflammatory disease of the airways that is often reversible. Sleep disorders in patients with asthma stems from nocturnal dyspnea and wakefulness, due to uncontrolled nighttime symptoms of asthma and drug-induced insomnia, ${ }^{2}$ as well as allergic rhinitis causing sleep impairment. Severe asthma has been associated with OSA and CPAP has primarily shown effectiveness in symptomatic control. ${ }^{3}$

- Interstitial lung disease: Individuals with interstitial lung disease develop progressive hypoxia due to inflammation and fibrosis of the lung parenchyma, developing desaturations during sleep, with or without concurrent apnea. Overall, there is worsened sleep architecture and quality in these patients. Some nonrespiratory disturbances include increased PLMD, RLS, and GERD in IPF and scleroderma patients. ${ }^{4}$ The association between severity of nocturnal desaturations and pulmonary hypertension also applies in ILD subjects. ${ }^{5}$

- Metabolic syndrome: This is characterized by a group of metabolic disorders including central obesity, impaired fasting glucose, dyslipidemia (hypertriglyceridemia and low high-

( The Author(s). 2019 Open Access This article is distributed under the terms of the Creative Commons Attribution 4.0 International License (https://creativecommons. org/licenses/by-nc/4.0/), which permits unrestricted use, distribution, and non-commercial reproduction in any medium, provided you give appropriate credit to the original author(s) and the source, provide a link to the Creative Commons license, and indicate if changes were made. The Creative Commons Public Domain Dedication waiver (http://creativecommons.org/publicdomain/zero/1.0/) applies to the data made available in this article, unless otherwise stated. 
density lipoprotein), and hypertension, resulting in overall increased risk of cardiovascular mortality. There is an increasing association between OSA and metabolic syndrome. Specifically, obesity, with predilection for fat deposition in upper airway, tongue, and abdomen predispose to OSA. ${ }^{6}$

- Chronic kidney disease (CKD): Multifactorial mechanisms of sleep fragmentation. The mechanism of 'rostral fluid shift' in a reclined position, where excess fluid accumulates in the neck leading to upper airway restriction and collapse, ${ }^{7}$ is seen in CKD patients with OSA. CPAP would be the first line treatment in this situation. Nocturnal melatonin levels in ESRD patients undergoing hemodialysis were significantly lower than their controls. ${ }^{8}$ This supported the significant role of melatonin, a sleep-wake circadian rhythm hormone secreted by the pineal gland, in forming the sleep architecture in CKD patients.

- Neuromuscular disorders (NMD): Since the diaphragm assumes all functions of respiration, neurogenic or neuromuscular diaphragmatic dysfunction is associated with sleep disorders, which are seen to typically precede diurnal respiratory failure in NMD patients. In such patients, positive airway pressure ventilation improves the quality of their sleep.

- Fibromyalgia (FM): It is a chronic pain disorder characterized by diffuse musculoskeletal pain and fatigue. The primary sleep disorder described here is a nonrestorative sleep, with alpha delta sleep noted on polysomnography. This indirectly results in poor sleep quality and quantity, increasing the risk of medical and psychiatric disease. ${ }^{9}$ Management includes cognitive behavioral therapy, exercise therapy, and use of neuropathic pain medications including amitriptyline.

- Eye disorders and OSA: In a review, Huon et al. have noted the increased association of glaucoma, retinal vein occlusion and floppy eyelid syndrome in subjects with OSA relative to non-OSA. Conversely, the odds of having OSA in patients with glaucoma, nonarteritic anterior ischemic optic neuropathy (NAION) and the central serous chorioretinopathy (CSR) group. Screening for OSA should be considered in patients with these eye disorders. ${ }^{10}$ As our patient population ages and survival in chronic disorders improves with therapeutic advances, maintaining a robust sleep/wake cycle remains critical. As we add medications or consider therapies in our current patient population with multiple comorbidities, the effect of our interventions on sleep should be kept in mind. Conversely, treating sleep disorders may impact breathing and mask the alerting signs of hypoglycemia. The dangers and impact of polypharmacy should always be considered.

\section{Pearl of Wisdom}

- Treatment of sleep disorders are particularly challenging in the setting of other chronic medical conditions. A balanced view of the risks and benefits should be adopted to preserve quality of life.

- Sleep disruption is frequently multifactorial and a systematic approach addressing sleep hygiene, circadian rhythm disorders, sleep disordered breathing, insomnia and restless legs would be most productive.

- Sleep-disordered breathing is often severe and may also require combined modalities for therapy - CPAP, oxygen, choice of sleeping pill.

- Medications given for medical disorders often affect sleep and can cause both sleep disruption and daytime sleepiness.

\section{References}

1. Flenley DC. Sleep in chronic obstructive lung disease. Clin Chest Med. 1985;6:651-661[PMID]:2935359.

2. Desager KN, Nelen V, et al. Sleep disturbances and daytime symptoms in wheezing school- aged children. J Sleep Res. 2005;14(1):77-82.

3. Ciftci TU, Guven SF, et al. Effects of nasal continuous positive airway pressure in uncontrolled nocturnal asthmatic patients with obstructive sleep apnoea syndrome. Resp Med. 2005;99(5):529-534.

4. Mermigkis C, Chapman J, et al. Sleep-related breathing disorders in patients with idiopathic pulmonary brosis. Lung. 2007;185:173-178 [PMID: 17436039 DOI: 10.1007/s00408-007-9004-3].

5. Miyahara $Y$, Miyahara $Y$, et al. Monitoring of nocturnal oxygen desaturation using pulse oximeter and apnomonitor in patients with chronic pulmonary disease. Respiration. 1995;62:348-352 [PMID: 8552867].

6. Schwab RJ, Pasirstein M, et al. Identification of upper airway anatomic risk factors for obstructive sleep apnea with volu- metric magnetic resonance imaging. Am J Respir Crit Care Med. 2003;168(5):522-530.

7. Elias RM, Bradley TD, et al. Rostral overnight fluid shift in end-stage renal disease: relationship with obstructive sleep apnea. Nephrol Dial Transplant. 2012;27:1569-1573 [PMID: 22058175 DOI: 10.1093/ ndt/gfr605].

8. Mehta R, Drawz PE. Is nocturnal blood pressure reduction the secret to reducing the rate of progression of hypertensive chronic kidney disease? Curr Hypertens Rep. 2011;13:378-385 [PMID: 21710375 DOI: 10.1007/s11906-011-0217-8].

9. Krystal AD. Sleep and psychiatric disorders: future directions.Psychiatr Clin North Am. 2006;29:1115-1130.

10. Huon LK, Liu SY, Camacho M, Guillemault C. The association between ophthalmic disease and obstructive sleep apnea: a systematic review and meta analysis. Sleep Breath. 2016;20(4):1145-1154. 